\title{
Ternary Constant Weight Codes
}

\author{
Patric R. J. Östergård* \\ Department of Computer Science \\ and Engineering \\ Helsinki University of Technology \\ P.O. Box 5400, 02015 HUT, Finland \\ patric.ostergard@hut.fi \\ Mattias Svanström ${ }^{\dagger}$ \\ Department of Electrical Engineering \\ Linköpings universitet \\ 58183 Linköping, Sweden \\ mattias@isy.liu.se
}

Submitted: October 1, 2002; Accepted: October 15, 2002.

MR Subject Classifications: 94B25, 05B40

\begin{abstract}
Let $A_{3}(n, d, w)$ denote the maximum cardinality of a ternary code with length $n$, minimum distance $d$, and constant Hamming weight $w$. Methods for proving upper and lower bounds on $A_{3}(n, d, w)$ are presented, and a table of exact values and bounds in the range $n \leq 10$ is given.
\end{abstract}

Keywords: bounds on codes, constant weight code, error-correcting code, ternary code.

\section{Introduction}

Constant weight codes constitute an important class of error-correcting codes [17]. Binary constant weight codes have therefore been thoroughly studied with the focus of attention on the function $A(n, d, w)$, which denotes the maximum cardinality of a binary code of length $n$, minimum distance $d$, and constant weight $w$. Extensive results on upper and lower bounds on $A(n, d, w)$ are presented in [1] and [7], respectively.

*Supported in part by the Academy of Finland under grant 100500.

${ }^{\dagger}$ Supported by the Swedish Research Council for Engineering Sciences under grant 271-97-532. 
When studying nonbinary constant weight codes, one may either prescribe the complete weight or the Hamming weight of the codes. Codes with given complete weight are called constant-composition codes. Ternary constant-composition codes are studied in [25]. The maximum size of a ternary code with minimum distance $d$ and complete weight enumerator of the form $A_{w_{0}, w_{1}, w_{2}} z_{0}^{w_{0}} z_{1}^{w_{1}} z_{2}^{w_{2}}$ (so $n=w_{0}+w_{1}+w_{2}$ ), where $A_{w_{0}, w_{1}, w_{2}}$ is the number of codewords with the given composition, is denoted by $A_{3}\left(n, d,\left[w_{0}, w_{1}, w_{2}\right]\right)$.

In this paper, we study ternary codes with given Hamming weight, and let $A_{q}(n, d, w)$ denote the maximum size of a $q$-ary code of length $n$, minimum distance $d$, and constant Hamming weight $w$ (where the index $q$ may be omitted in the binary case). A code of size $M$ for given values of $q, n, d$, and $w$ is called an $(n, d, w ; M)_{q}$ code. An $\left(n, d, w ; A_{q}(n, d, w)\right)_{q}$ code is said to be optimal. The function $A_{3}(n, d, w)$ has until recently received only limited attention. Published work on this topic includes $[4,10,12$, $11,16,22,23,26,27]$ and the thesis [24] of the second author.

The paper is organized as follows. In Section 2 some basic results on ternary constant weight codes are presented. Methods for obtaining upper bounds on $A_{3}(n, d, w)$ are considered in Section 3. In Section 4 constructions of ternary constant weight codes, which lead to lower bounds on $A_{3}(n, d, w)$, are discussed. A table of $A_{3}(n, d, w)$ for $n \leq 10$ is presented in Section 5.

\section{Preliminaries}

The coordinate values of ternary codes are without loss of generality $\{0,1,2\}$. If desired, these may be taken as elements of $F_{3}$, the Galois field of order 3. None of the constructions in this work, however, require any algebraic structure of this underlying set.

The rest of this section is devoted to several special cases where we can determine the exact value of $A_{3}(n, d, w)$. Let $A_{q}(n, d)$ denote the maximum size of a $q$-ary code (without weight restrictions) of length $n$ and minimum distance $d$, where the index may be omitted when $q=2$. Tables of $A_{2}(n, d)$ and $A_{3}(n, d)$ can be found in [2] and [6], respectively. The proof of the following theorem is obvious and is omitted.

Theorem $1 A_{q}(n, d, n)=A_{q-1}(n, d)$.

By Theorem $1, A_{3}(n, d, n)=A(n, d)$. Since binary unrestricted codes have been thoroughly studied in the literature - see, for example, [7] - we assume that $w<n$ in the sequel. In the following proofs, the concept of support plays a central role. The support of a codeword is the set of coordinates with non-zero values. The following theorem is also proved in [12]; we give an alternative proof.

Theorem $2 \quad A_{q}(n, 2, w)=\left(\begin{array}{l}n \\ w\end{array}\right)(q-1)^{w-1}$.

Proof: There are $\left(\begin{array}{l}n \\ w\end{array}\right)$ possible supports for codewords with weight $w$. Two words with different support are clearly at distance at least 2 from each other. For a given support, the maximum number of codewords with minimum distance 2 is $A_{q}(w, 2, w)=A_{q-1}(w, 2)$ 
(by Theorem 1), and the proof is completed as $A_{q}(w, 2)=q^{w-1}$.

For small values of $M$, we are able to determine for what parameters $A_{3}(n, d, w)=M$. (Trivially, $A_{3}(n, d, w) \geq 1$ for any parameters.) The following lemmas will be useful in proving such results. The proof of the first lemma is obvious and is omitted.

Lemma 3 If $A_{3}(n, d, w) \geq 2$, then $d \leq 2 w$ and $d \leq n$.

Lemma $4 A_{3}(n, d, w) \leq A(n, 2(d-w), w)$ if $d / 2 \leq w \leq d$.

Proof: If the supports of two codewords of a ternary code attaining $A_{3}(n, d, w)$ intersect in $t$ coordinates, then they are at distance at most $(w-t)+t+(w-t)=2 w-t$ from each other, and we get $2 w-t \geq d$, so $t \leq 2 w-d$. Therefore, the supports correspond to a binary code with constant weight $w$ and minimum distance at least $2 w-2(2 w-d)=2 d-2 w$, which has size at most $A(n, 2(d-w), w)$.

Lemma 4 is strongest when $w$ is close to $d / 2$. At the other extreme, when $w=d$, the implication of the lemma is trivial.

Theorem $5 A_{3}(n, d, w) \geq 2$ exactly when $d \leq \min \{2 w, n\}$. $A_{3}(n, d, w) \geq 3$ exactly when $d \leq \min \{2 w, n / 3+w, 5 n / 3-w\}$.

Proof: By Lemma 3, the given conditions for $A_{3}(n, d, w) \geq 2$ are necessary. Their sufficiency is proved by two words whose supports intersect in $2 w-d$ coordinates and have different values in these coordinates.

It is known that $A(n, d, w) \geq 3$ exactly when $n \geq 3 d / 2$ and $n \geq w+d / 2$ (see [7, p. 1338]). By Lemma 4, necessary conditions for $A_{3}(n, d, w) \geq 3$ are therefore $n \geq d$ and $n \geq 3(d-w)$. When $w \leq 2 n / 3$, the latter condition $(d \leq n / 3+w)$ is sufficient, which can be seen by finding a transformation from a binary constant weight code as follows.

Note that when $w \leq 2 n / 3$ we can (if necessary) always transform a binary constant weight code with three words and a given minimum distance into another binary constant weight code with the same parameters (but possibly larger minimum distance) such that there is no coordinate where all words have a 1 . (Namely, we can transpose a 1 in such a coordinate with a 0 in a coordinate with at most one $1-$ which exists since the average number of $1 \mathrm{~s}$ in the coordinates is $3 w / n \leq 2 n / n=2$. This procedure is repeated until there is no coordinate with three 1s.) A code with this property is then made ternary by changing one of the $1 \mathrm{~s}$ in a coordinate with two 1 s into a 2.

We now consider $w>2 n / 3$ and count the distances between all pairs of three given ternary words in two ways. The pairwise distances are at least $d$, so a lower bound for the sum is $3 d$. Denote the number of coordinates with one, two, and three nonzero values by $a, b$, and $c$, respectively (w.l.o.g, we may assume that there are no coordinates with only one value and that with two nonzero values, these are different). Then we have 


$$
\left\{\begin{array}{l}
a+2 b+3 c=3 w \\
a+b+c=n
\end{array}\right.
$$

and the sum of distances is $2 a+3 b+2 c$, which equals $5 n-3 w-2 a$ using (1), and by combining this and the earlier result we get $3 d \leq 5 n-3 w-2 a$, so $3 d \leq 5 n-3 w$.

A code can be constructed in the following way to prove that $3 d \leq 5 n-3 w$ is sufficient when $w>2 n / 3$. Let the $n-w$ coordinates of 0 s in the three words be nonoverlapping. Then there are $3 w-2 n$ coordinates where all words are nonzero, and the distance between two words in these coordinates can be made at least $\lfloor 2(3 w-2 n) / 3\rfloor$ as $A(n,\lfloor 2 n / 3\rfloor) \geq 3$. The distance between two words can thus be made at least $\lfloor 2(3 w-2 n) / 3\rfloor+3(n-w)=\lfloor 5 n / 3\rfloor-w$, and as the parameters are integers, $3 d \leq 5 n-3 w$ (that is, $d \leq 5 n / 3-w)$.

Corollary $6 A_{3}(n, d, w)=1$ exactly when $d>\min \{2 w, n\} . A_{3}(n, d, w)=2$ exactly when $\min \{n / 3+w, 5 n / 3-w\}<d \leq \min \{2 w, n\}$.

Throughout the results shown so far, the obvious condition $d \leq 2 w$ has been present for nontrivial codes. Another family of optimal codes is obtained when $d=2 w$.

Theorem $7 \quad A_{3}(n, 2 w, w)=\lfloor n / w\rfloor$.

Proof: With $d=2 w$, no codewords can have overlapping supports. The maximum number of codewords with this property is $\lfloor n / w\rfloor$.

For $d=2 w-1$, we have the following result.

Theorem $8 A_{3}(n, 2 w-1, w)=\max \left\{M \mid n \geq\lceil M w / 2\rceil+\max \left\{\lfloor M w / 2\rfloor-\left(\begin{array}{c}M \\ 2\end{array}\right), 0\right\}\right\}$.

Proof: With minimum distance $d=2 w-1$, the supports of two words can have at most one common coordinate. Moreover, no three codewords may have a common coordinate in their supports, since then at least two of these would have the same value in that coordinate and mutual distance at most $2 w-2$. It is enough to study binary constant weight codes with the same supports, since the required ternary code can then always be obtained by changing a 1 into a 2 in a coordinate with two nonzero values.

Hence we consider binary codes with constant weight $w$ and the properties that two words have at most one common 1 and no three words have a common 1 . This can be viewed as an incidence matrix of a graph - the words correspond to vertices and the the coordinates to edges - with vertex degrees at most $w$ (ignoring the loops).

We fix the number of codewords, $M$, that is, the number of vertices in the corresponding graph, and calculate the smallest length $n$ for which a corresponding code exists (then it also exists for any larger $n$ ). To minimize $n$, we have to maximize the number of coordinates with two nonzero values, that is, the number of edges in a graph with vertex degrees at most $k=\min \{w, M-1\}$. 
The number of edges in a graph with $M$ vertices and vertex degrees at most $k$ is, by a direct counting argument, at most $\lfloor M k / 2\rfloor$ and we shall see that there are graphs that attain this value. This can be done in several ways; we use one-factorizations. If $M$ is even, the complete graph $K_{M}$ has a one-factorization [28, Theorem 3.2], so we take $k$ one-factors. If $M$ is odd, start with $k$ one-factors of a graph with $M-1$ vertices, delete $\lfloor k / 2\rfloor$ edges from one of the factors, introduce a new vertex, and connect the new vertex to all vertices incident to the deleted edges [28, p. 23].

Now if $w \leq M-1$, the minimum value of $n$ is $\lceil M w / 2\rceil$. If $w>M-1$, the code will have $M w-2\left(\begin{array}{c}M \\ 2\end{array}\right)$ coordinates with one nonzero value in addition to the $\left(\begin{array}{c}M \\ 2\end{array}\right)$ coordinates with two values, so the length is at least $M w-\left(\begin{array}{c}M \\ 2\end{array}\right)$. By combining these results, the theorem is proved.

\section{$3 \quad$ Upper Bounds}

Whereas lower bounds follow from explicitly constructed codes, many of which can be verified even by hand, several of the best known upper bounds have been obtained computationally and require as large effort for verification as for the original proof. In this section, the methods that are used to produce the upper bounds of the main table are briefly discussed; it is an interesting open research problem to try to develop even more sophisticated bounds using, for example, the ideas in [1].

\subsection{Johnson-Type Bounds}

Johnson [14] presented bounds for binary error-correcting codes. Analogous bounds have later been developed for many other types of error-correcting codes, including ternary constant weight codes. Bounds that actually are useful for producing both upper and lower bounds are given in the following two theorems [24].

\section{Theorem 9}

$$
A_{q}(n, d, w) \leq \frac{n}{n-w} A_{q}(n-1, d, w)
$$

Proof: An optimal code attaining $A_{q}(n, d, w)$ must have a coordinate with at least $(n-w) A_{q}(n, d, w) / n 0 \mathrm{~s}$. By shortening the code with respect to the $0 \mathrm{~s}$ in this coordinate and deleting the coordinate, we get a code with at most $A_{q}(n-1, d, w)$ words.

If we instead shorten a code with respect to a nonzero value, we get another similar bound. The proof mimics that of Theorem 9 and is omitted.

\section{Theorem 10}

$$
A_{q}(n, d, w) \leq \frac{n(q-1)}{w} A_{q}(n-1, d, w-1)
$$


Note that in using Theorems 9 and 10 and a lower bound on $A_{3}(n, d, w)$, instead of applying these averaging formulas, we may dissect the code to see if there are even larger subcodes.

The next bound is obtained by counting the distances between words in two different ways: for all pairs of words and coordinatewise. Actually, we used this technique already in Section 2. A slightly weaker bound for $q$-ary constant weight codes is presented in [12]. The ternary version of our main result appears in [24].

Lemma 11 Fill an $M \times n$ matrix $A$ with entries $a_{i, j}(1 \leq i \leq n, 1 \leq j \leq M)$ from $\{0,1, \ldots, q-1\}$, a of which are 0 . Then $\sum_{i=1}^{n} \sum_{j=1}^{M} \sum_{j^{\prime}=j+1}^{M} d\left(a_{i, j}, a_{i, j^{\prime}}\right)$ achieves its maximum value when the values of entries are evenly distributed: $\lfloor a / n\rfloor$ or $\lceil a / n\rceil$ os in each column; if there are $b$ nonzero entries in a column, then there are $\lfloor b /(q-1)\rfloor$ or $\lceil b /(q-1)\rceil$ of each of the nonzero values in this column.

Proof: Let $n_{i, j}$ denote the number of entries $j$ in column $i$. If a given matrix has $a 0 \mathrm{~s}$, then so has the matrix after a replacement of a nonzero value by another nonzero value. By [5, Lemma 1], in a column $i$ with $b$ nonzero entries, the maximum sum of the distances between all pairs of entries is achieved when $n_{i, j}=\lfloor(b+j-1) /(q-1)\rfloor$, so we assume from now on that the matrix has this property.

Assume that $n_{i, 0}-n_{i^{\prime}, 0}>1$ for two columns $i$ and $i^{\prime}$. Then if one 0 in column $i$ is replaced by a 1 and one entry $(q-1)$ in column $i^{\prime}$ is replaced by a 0 , we get another matrix with the stipulated properties whose objective function differs from that of the old matrix by

$$
\begin{aligned}
& n_{i, 0}-1-n_{i, 1}-n_{i^{\prime}, 0}+\left(n_{i^{\prime}, 1}-1\right)=\left(n_{i, 0}-n_{i^{\prime}, 0}\right)+\left(n_{i^{\prime}, q-1}-n_{i, 1}\right)-2 \\
& \geq 2+1-2>0 .
\end{aligned}
$$

Hence we may assume that the values are evenly distributed as given by the lemma.

We define $k=\lfloor M w / n\rfloor$ and $t=M w-n k$.

Theorem 12 If there exists an $(n, d, w ; M)_{q}$ code, then

$$
M(M-1) d \leq 2 t \sum_{i=0}^{q-2} \sum_{j=i+1}^{q-1} M_{i} M_{j}+2(n-t) \sum_{i=0}^{q-2} \sum_{j=i+1}^{q-1} M_{i}^{\prime} M_{j}^{\prime},
$$

where $M_{0}=M-k-1, M_{0}^{\prime}=M-k, M_{i}=\lfloor(k+i) /(q-1)\rfloor$, and $M_{i}^{\prime}=\lfloor(k+i-1) /(q-1)\rfloor$.

Proof: In a code with the given parameters, we sum the distances between all ordered pairs of codewords in two different ways. One way is given by Lemma 11; the sum in this case is bounded from above by the expression on the right side of the inequality in this theorem. On the other hand, as the code has minimum distance $d$, the sum of distances is bounded from below by $M(M-1) d$, and the result follows.

For ternary codes, we get the following corollary. 
Corollary 13 If there exists an $(n, d, w ; M)_{3}$ code, then for even $k$,

$$
\left(\frac{3 k^{2}}{2}-2 k\right) n+3 k t \leq M(M-1)(2 w-d)
$$

and for odd $k$,

$$
\left(\frac{3 k^{2}}{2}-2 k+\frac{1}{2}\right) n+(3 k-1) t \leq M(M-1)(2 w-d) .
$$

Example 1. For the parameters $n=6, d=5$, and $w=4$, [12, Lemma 5.1] gives that $A_{3}(6,5,4) \leq 5$, whereas $A_{3}(6,5,4) \leq 4$ by Theorem 12 (or Corollary 13). For example, to see that a $(6,5,4 ; 5)_{3}$ code does not exist, we have $k=3, t=2, M_{0}=1, M_{1}=2$, $M_{2}=2, M_{0}^{\prime}=2, M_{1}^{\prime}=1$, and $M_{2}^{\prime}=2$, and evaluating the expression in Theorem 12 gives $100 \leq 4 \cdot(2+2+4)+6(2+4+2)=80$, a contradiction.

\subsection{Families of Perfect and Optimal Codes}

As mentioned earlier, the results presented in this work are mainly those that turn out to be good in the sense that they can be used to produce entries in the record table to be presented in the final section. An alternative way of evaluating constructions is to see whether they produce families of codes that are optimal or asymptotically optimal as the length tends to infinity. Several such families have been published in the literature.

Generalized Steiner triple systems have recently received a lot of attention; see, for example, $[3,8,10]$. A generalized Steiner triple system is a $q$-ary code of constant weight 3 and minimum distance 3 , and with the property that all words of weight 2 are at distance 1 from a codeword. These are of great interest as they form optimal constant weight codes. In particular, the ternary case has been studied and settled [10].

Theorem $14 A_{3}(n, 3,3)=2 n(n-1) / 3$ for $n \equiv 0,1(\bmod 3), n \geq 4, n \neq 6$.

Optimality of the codes attaining the bound in Theorem 14 follows from Theorems 8 and 10. Alternatively, one may use the fact that each codeword is at distance 1 from three words of weight 2 ; there are $2 n(n-1)$ words of weight 2 , none of which may be at distance 1 from two codewords. Also the case $n \equiv 2(\bmod 3)$ has been settled [29].

Theorem $15 A_{3}(n, 3,3)=2 n(n-1) / 3-4 / 3$ for $n \equiv 2(\bmod 3), n \geq 5$.

Note that Theorem 15 actually differs slightly from the result in [29], where it is claimed that the result holds only for $n \geq 8$ and does not hold for $n=5$. The computer search carried out in [29] is obviously in error, since a $(5,3,3 ; 12)_{3}$ code is known to exist [4]. Such a code with a particular structure is given later in Table 3.

Jacobsthal matrices are used in [25] to produce good constant-composition codes; they can also be used to obtain optimal ternary constant weight codes [24]. Let $p$ be an odd 
prime. Consider the Galois field $F_{p^{m}}$ with elements $\alpha_{1}, \alpha_{2}, \ldots, \alpha_{p^{m}}$ and let $S$ be the set of nonzero square elements

$$
S=\left\{\alpha_{i}^{2}: \alpha_{i} \in F_{p^{m}}, \alpha_{i} \neq 0\right\} .
$$

A $p^{m} \times p^{m}$ Jacobsthal matrix $Q=\left(q_{i j}\right)$ is defined by

$$
q_{i j}=\left\{\begin{aligned}
0 & \text { if } \alpha_{i}=\alpha_{j}, \\
1 & \text { if } \alpha_{i}-\alpha_{j} \in S, \\
-1 & \text { otherwise. }
\end{aligned}\right.
$$

Theorem 16 If $p \geq 3$ is a prime and $m \geq 1$, then $A_{3}\left(p^{m}+1,\left(p^{m}+3\right) / 2, p^{m}\right)=2 p^{m}+2$.

Proof: Take a $p^{m} \times p^{m}$ Jacobsthal matrix $Q$ with entries from $\{-1,0,1\}$ (which exists with the given restrictions on the parameters [17, p. 47]) and map the entries to $\{0,1,2\}$ by $-1 \rightarrow 2,0 \rightarrow 0,1 \rightarrow 1$. Take the rows of this matrix, add one coordinate with value 1 , and call the resulting code $C_{1}$. Let $C_{2}$ be the code obtained by interchanging the values 1 and 2 in all coordinates of $C_{1}$.

We now claim that $C=C_{1} \cup C_{2} \cup\{011 \ldots 1,022 \ldots 2\}$ proves that $A_{3}\left(p^{m}+1,\left(p^{m}+\right.\right.$ $\left.3) / 2, p^{m}\right) \geq 2 p^{m}+2$. The only nontrivial part of this proof is to show that the minimum distance is at least $\left(p^{m}+3\right) / 2$. The inner product of two distinct rows of $Q$ is -1 by $\left[17\right.$, p. 47 , Lemma 7]. As exactly $p^{m}-2$ of the coordinates have nonzero values in both of these rows, we find that they coincide in $\left(p^{m}-3\right) / 2$ and differ in $\left(p^{m}-1\right) / 2$ of these coordinates. The distance between two words in $C_{1}$ (or $\left.C_{2}\right)$ is therefore $\left(p^{m}-1\right) / 2+2$. The analysis above shows that if $C_{2}$ is obtained by interchanging 1 and 2 in $C_{1}$, then the distance between a word in $C_{1}$ and another in $C_{2}$ is $1+\left(p^{m}-3\right) / 2+2$. The distance criterion also holds for the two supplementary words of $C$ as there are $\left(p^{m}-1\right) / 2$ values of both -1 and 1 in each row of $Q$.

The theorem now follows by using $A_{3}\left(p^{m},\left(p^{m}+3\right) / 2, p^{m}-1\right) \leq p^{m}$ (from Theorem 12) and Theorem 10.

The result $A_{3}\left(p^{m},\left(p^{m}+3\right) / 2, p^{m}-1\right)=p^{m}$ from [12] is then a corollary of this result (by using Theorems 10 and 12).

Infinite families of codes with $w=3$ that are asymptotically optimal are presented in [22] and generalized in [11].

Of particular interest is the following recently discovered [23] family of perfect oneerror-correcting constant weight codes. A code $C$ in a given space is said to be a perfect $t$-error-correcting code if all words in the space are at distance at most $t$ from exactly one word in $C$.

Theorem 17 For $r \geq 2, A_{3}\left(2^{r}, 3,2^{r}-1\right)=2^{2^{r}-1}$ and corresponding codes are perfect.

After having seen some of the preliminary results of [23], the authors of [16] published an alternative proof of Theorem 17 and also showed that these codes and the perfect binary codes of length $2^{r}-1, r \geq 2$ (which are ternary constant weight codes with $w=n$ ) are the only perfect ternary constant weight codes with $d=3$. 


\subsection{Exhaustive Computer Search}

A known upper bound $A_{q}(n, d, w) \leq M$ may be improved after an exhaustive computer search for a code with these parameters and size $M$. This search can in fact be conveniently described as a search for a clique of size $M$ in the following graph. Consider the graph where the vertex set corresponds to the words of length $n$ and Hamming weight $w$ and two vertices are joined by an edge if the Hamming distance between the corresponding words is greater than or equal to $d$.

With a maximum clique algorithm, we would find the exact value of $A_{q}(n, d, w)$ but this direct approach is computationally feasible only for very small parameters. We may then perhaps relax the goal and just try to lower the upper bound. In any case, to speed up the search, it is essential to handle the large automorphism group of the constructed graph. This may be done in the following way by utilizing the Johnson-type bounds (Theorems 9 and 10) and removing equivalent copies of partial codes.

Two $q$-ary constant weight codes are said to be equivalent if one can be mapped onto the other by permuting the coordinates and permuting the nonzero values in each coordinate. Such transformations are distance-preserving and weight-preserving (and constitute the stabilizer of the all-zero word in the group of distance-preserving transformations of unrestricted codes). An equivalence transformation that maps a code onto itself is an automorphism. The set of all automorphisms of a code constitutes the (full) automorphism group of the code.

As in proving Theorems 9 and 10, we find that an $(n, d, w ; M)_{q}$ code can be shortened to get $\left(n-1, d, w ; M^{\prime}\right)_{q}$ and $\left(n-1, d, w-1 ; M^{\prime \prime}\right)_{q}$ subcodes, where

$$
M^{\prime} \geq \frac{n-w}{n} M, M^{\prime \prime} \geq \frac{w}{n(q-1)} M .
$$

Therefore, we may construct a code $C$ by classifying all such subcodes (for one of these two alternatives), and then use the clique-finding approach to find the rest of the words in $C$. This computation can be done recursively as the following example shows.

Example 2. It was known to us that $9 \leq A_{3}(7,5,5) \leq 10$, so we wanted to find out whether there exists a $(7,5,5 ; 10)_{3}$ code. By using $(2)$, there must be a $\left(6,5,5 ; M^{\prime}\right)_{3}$ subcode with $M^{\prime} \geq 3$. There is a unique such code (for which $M^{\prime}=3$ ), which can be constructed by hand or by lengthening all $(5,5,5 ; 1)_{3}$ and $(5,5,5 ; 2)_{3}$ codes (which are, up to equivalence, $\{11111\}$ and $\{11111,22222\}$, respectively). Since the unique $(6,5,5 ; 3)_{3}$ code cannot be lengthened to a $(7,5,5 ; 10)_{3}$ code (this is preferably done by computer), we find that $A_{3}(7,5,5)=9$.

To prove equivalence of codes, we have mapped codes to graphs as in [25] (with a mapping suiting this class of codes and definition of equivalence) and used the graph isomorphism program nauty [18].

In four cases when we tried to improve the best known upper bound, we encountered a new code, thereby proving an exact value that is greater than the previously best known lower bound. These four codes are among those listed in Table 1. 


\begin{tabular}{ll}
\hline Bound & Codewords \\
\hline$A_{3}(8,5,4) \geq 13$ & $11110000,01021100,00212200,21000210,20022020$, \\
& $10200120,02201010,20101001,20010102,12002002$, \\
& $02010021,00120012,00002111$ \\
$A_{3}(8,5,5) \geq 18$ & $11110001,01021101,00212201,12000111,10022021$, \\
& $02201021,22200202,22122000,21100120,21020012$, \\
& $20012110,12011200,11202010,10120210,10102102$, \\
& $01002222,00220122,00111012$ \\
$A_{3}(8,5,6) \geq 18$ & $11111001,22220101,20012111,12022021,02101221$, \\
& $01210211,22110012,21201110,21002222,20211202$, \\
& $12102102,12011210,11222200,11020112,10121120$, \\
& $02212120,01221022,00122212$ \\
$A_{3}(9,5,4) \geq 19$ & $111100000,010211000,002122000,200101100$, \\
& $120020100,021002200,210002010,200220020$, \\
& $102001020,022010010,001200110,000012120$, \\
& $201010001,100202002,100000211,020100021$, \\
& $012000102,000110202,000021012$ \\
& $1111111000,1001222110,0220211210,0212120120$, \\
& $2210022011,2201201022,2100110112,2002121201$, \\
$A_{3}(10,7,7) \geq 1221020202,1022210021,1010102222,0122202102$, \\
& 0101012221 \\
\hline
\end{tabular}

Table 1: Explicitly listed codes. 


\section{Lower Bounds}

Lower bounds are constructive and follow from explicit constructions of constant weight codes. Two main constructions - both of which are computer-aided - are presented in this section. For more constructions that can be used to obtain good ternary constant weight codes, see [24]. Various constructions were, of course, also discussed earlier in this paper along with results on exact values of $A_{3}(n, d, w)$.

Trivially, as a constant-composition code is also a constant weight code, we have that

$$
A_{q}\left(n, d, w_{1}+w_{2}\right) \geq A_{q}\left(n, d,\left[w_{0}, w_{1}, w_{2}\right]\right) .
$$

In using this inequality applied to bounds on constant-composition codes, it is useful to note that $A_{q}\left(n, d,\left[w_{0}, w_{1}, w_{2}\right]\right)=A_{q}\left(n, d,\left[w_{f(0)}, w_{f(1)}, w_{f(2)}\right]\right)$, for any bijection $f:\{0,1,2\} \rightarrow\{0,1,2\}$.

\subsection{Lexicographic Codes}

As some of the lower bounds on binary constant weight codes in [7] come from lexicographic codes, it is no surprise that some of the best known ternary constant weight codes are also lexicographic.

A ternary constant weight lexicographic code (lexicode) is obtained in the following way. We start by prescribing the parameters of the code $-n, d$, and $w$-and a so-called seed: a (possibly empty) set of words that belong to the code. Then, with a given order of the words, we go through all words in this order and add words to the code whenever they are at distance at least $d$ from all previously accepted words.

In this study, some lower bounds are obtained with a new type of lexicographic codes, which we call lexicographic codes with offset. These codes are constructed as regular lexicodes, but instead of starting from the lexicographically first word in the space, we start from a given word (called offset vector) and proceed cyclically through all words. The bounds obtained in this way are presented in Table 2. Some other record-breaking codes are also achieved by lexicodes; cf. [4].

\begin{tabular}{ll}
\hline Bound & Offset \\
\hline$A_{3}(6,5,4) \geq 4$ & - \\
$A_{3}(10,7,5) \geq 8$ & 0012100011 \\
$A_{3}(10,7,6) \geq 11$ & 0011221100 \\
\hline
\end{tabular}

Table 2: Lexicographic codes

\subsection{Constant Weight Codes from Unrestricted Codes}

If we take a (linear or nonlinear) unrestricted code with minimum distance $d$, then its subcode formed by codewords with a given weight clearly has minimum distance at least 
d. There is, of course, a wide variety of codes whose subcodes one could study in the search for good constant weight codes. It is perhaps no surprise that perfect codes are good candidates.

The complete weight enumerator of the ternary Golay code (with minimum distance 5) and the extended ternary Golay code (with minimum distance 6) are, respectively,

$$
\begin{aligned}
W_{1}\left(z_{0}, z_{1}, z_{2}\right)= & z_{0}^{11}+z_{1}^{11}+z_{2}^{11}+ \\
& 11\left(z_{0}^{6} z_{1}^{5}+z_{0}^{5} z_{1}^{6}+z_{0}^{6} z_{2}^{5}+z_{0}^{5} z_{2}^{6}+z_{1}^{6} z_{2}^{5}+z_{1}^{5} z_{2}^{6}\right)+ \\
& 55\left(z_{0}^{6} z_{1}^{3} z_{2}^{2}+z_{0}^{6} z_{1}^{2} z_{2}^{3}+z_{0}^{3} z_{1}^{6} z_{2}^{2}+z_{0}^{3} z_{1}^{2} z_{2}^{6}+z_{0}^{2} z_{1}^{6} z_{2}^{3}+z_{0}^{2} z_{1}^{3} z_{2}^{6}\right)+ \\
& 110\left(z_{0}^{5} z_{1}^{3} z_{2}^{3}+z_{0}^{3} z_{1}^{5} z_{2}^{3}+z_{0}^{3} z_{1}^{3} z_{2}^{5}\right), \\
W_{2}\left(z_{0}, z_{1}, z_{2}\right)= & z_{0}^{12}+z_{1}^{12}+z_{2}^{12}+ \\
& 22\left(z_{0}^{6} z_{1}^{6}+z_{0}^{6} z_{1}^{6}+z_{0}^{6} z_{2}^{6}\right)+ \\
& 220\left(z_{0}^{6} z_{1}^{3} z_{2}^{3}+z_{0}^{3} z_{1}^{6} z_{2}^{3}+z_{0}^{3} z_{1}^{3} z_{2}^{6}\right) .
\end{aligned}
$$

From $W_{1}\left(z_{0}, z_{1}, z_{2}\right)$ we find that $A_{3}(11,5,5) \geq 132$ (which in fact turns out to be an exact value) and by using Theorem 10 , we find that $A_{3}(10,5,4) \geq 30$. From $W_{2}\left(z_{0}, z_{1}, z_{2}\right)$ we find that $A_{3}(12,6,6) \geq 264$ and $A_{3}(12,6,9) \geq 440$ (which also turn out to be exact values) and by using Theorems 9 and 10 to shorten the code twice, we arrive at $A_{3}(10,6,5) \geq 36$, $A_{3}(10,6,6) \geq 60, A_{3}(10,6,7) \geq 60$, and $A_{3}(10,6,8) \geq 45$.

Occasionally, optimal unrestricted codes can be constructed from constant weight codes. For example, by taking an $(7,4,5,32)_{3}$ code and one word of weight 0 or 1 , we find that $A_{3}(7,4) \geq 33$. Actually, we know [20] that $A_{3}(7,4)=33$, so this argument can then in fact be used to prove that $A_{3}(7,4,5)=32$. Analogously, we get upper bounds that together with constructions of corresponding codes show that $A_{3}(7,5,5)=9$ $\left(A_{3}(7,5)=10\right.$ from $\left.[27]\right)$ and $A_{3}(10,7,7)=13\left(A_{3}(10,7)=14\right.$ from $\left.[15]\right)$.

\subsection{Computer Search with Symmetries}

The approach presented in Section 3.3 does not impose any restrictions on the structure of codes. With restrictions, however, the search space can be reduced and even larger instances can be attacked. This approach can, of course, be successful only if there exist codes with the prescribed restrictions. One obvious restriction is to prescribe automorphisms (symmetries) of the codes one is searching for.

In this work, mainly cyclic and quasi-cyclic codes are considered. A cyclic code has an automorphism that cyclically permutes all coordinates. One may generalize this code family slightly to include codes for which the cyclic permutation does not act on all coordinates (and say that the code has one or more fixed coordinates). A quasi-cyclic codes has an automorphism that is a nontrivial subgroup of an aforementioned automorphism of a cyclic code.

In the search for the largest code with a prescribed automorphism group, we first compute all orbits of words, and reject those orbits in which there is a pair of words at 
distance less than $d$ from each other. The search problem can also here be presented as a clique problem, but now we have a graph where the vertices correspond to orbits and vertices indicate that all words in one orbit are at distance greater than or equal to $d$ from all words in the other orbit. Moreover, the vertices have integer weights - given by the number of words in the corresponding orbit - and hence we have an instance of the maximum-weight clique problem.

Whenever possible, we solved the instances of the maximum-weight clique problem using the program Cliquer; see [19, 21]. For instances that are too hard to solve exactly (we stopped the search if an instance could not be solved within a few hours), one may of course use any stochastic method of choice to find cliques with large weight; in this work, however, we did not proceed very far in this direction. In a few cases, the best known lower bound comes from an intermediate result of an interrupted run of the clique algorithm.

The codes obtained in this way are listed in Table 3. The leftmost coordinate of a codeword is numbered 1 and the rightmost is numbered $n$. The groups used and their generators are given in Table 4. The list of transitive permutation groups in [9] was extensively utilized in the search. The group $G^{\prime}$ is the nonabelian group of order 27 with presentation $\left\langle s, t: s^{9}=t^{3}=1, s t=t s^{4}\right\rangle$. The notation $p \times G$, where $p$ is an integer, is used in Table 3 to show that a group acts on $p k$ coordinates instead of $k$ coordinates (from Table 4), as described by the following example.

Example 3. Let $n=7$. Then $C_{2}$ is generated by $(12)(3)(4)(5)(6)(7), 2 \times C_{2}$ is generated by $(12)(34)(5)(6)(7)$, and $3 \times C_{2}$ is generated by $(12)(34)(56)(7)$.

\section{The Main Table}

The best known upper and lower bounds (and exact values when these coincide) for ternary constant weight codes of length $n \leq 10$ are displayed in Table 5 . Entries for $d>n$, which are 1 , are omitted to make the table more readable.

One reference is given for each upper and lower bound. However, references for entries that follow from Corollary 6 (whose values are 1 or 2 ) are omitted. Even if a bound can be obtained in several ways, only one reference is given. 


\begin{tabular}{|c|c|c|}
\hline$\overline{B o u n d}$ & Group & Orbit Representatives \\
\hline$\overline{A_{3}(5,3,3) \geq 12}$ & $2 \times C_{2}$ & $\begin{array}{l}\text { 12200, 20210, 11001, 20101, 00221, 22002, 01202, } \\
00112\end{array}$ \\
\hline$A_{3}(6,3,3) \geq 18$ & $2 \times C_{3}$ & 210100, 022100, 120200, 011200, 100110, 200220 \\
\hline$A_{3}(6,4,3) \geq 8$ & $C_{6}$ & 101200,202020 \\
\hline$A_{3}(7,3,4) \geq 60$ & $2 \times C_{3}$ & $\begin{array}{lllll}1121000, & 2212000, & 2201100, & 1022100, & 2011200, \\
1102200, & 0012110, & 0202210, & 2101001, & 1011001, \\
1202001, & 2022001, & 0021101, & 0012201, & 0221002, \\
0112002, & 1001102, & 0202102, & 0101202,2002202\end{array}$ \\
\hline$A_{3}(7,3,5) \geq 72$ & $C_{6}$ & 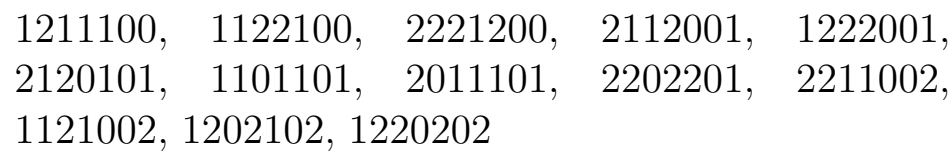 \\
\hline$A_{3}(7,4,3) \geq 14$ & $C_{7}$ & 1011000,2202000 \\
\hline$A_{3}(7,4,4) \geq 23$ & $C_{6}$ & 1221000, 2011100, 2202200, 2020201, 1012002 \\
\hline$A_{3}(7,4,5) \geq 32$ & $3 \times C_{2}$ & $\begin{array}{lllll}0212210, & 2211001, & 1110101, & 0222101, & 1021201, \\
2102201, & 2200111, & 0011111, & 0120211, & 2010221, \\
1122002, & 1201102, & 2012102, & 2220202, & 0111202, \\
1020112,2001212,1100222, & 0022222 & \end{array}$ \\
\hline$\geq 9$ & $2 \times C_{3}$ & 0122210, 2022101, 1101202 \\
\hline$\geq 161$ & $C_{7}$ & $\begin{array}{l}\text { 12221000, 21112000, 21210100, 12120100, } 22201100, \\
\text { 20122100, 22021200, 12110001, 21220001, 22101001, } \\
\text { 11021001, 11202001, 22012001, 20110101, 22020101, } \\
\text { 11210002, 22120002, 12011002, 20211002, 21022002, } \\
10122002,11020102,20220102\end{array}$ \\
\hline$A_{3}(8,3,6) \geq 168$ & $C_{8}$ & $\begin{array}{l}11121100,21212100,22111200,12221200,11112200, \\
21211010,21122010,12222010,22110110,21220110, \\
12011110,20121110,12102110,12210210,11120210, \\
11202210,20222210,11212020,12220120,11201120, \\
22022120,22202220\end{array}$ \\
\hline$A_{3}(8,4$, & $C_{8}$ & 10220000,20011000 \\
\hline$A_{3}(8,4$ & $C_{8}$ & $\begin{array}{l}21101000,12021000,10112000,10220100,22200200, \\
20202020\end{array}$ \\
\hline$A_{3}(8,4,5) \geq 72$ & $C_{8}$ & $\begin{array}{l}\text { 11121000, 22212000, 21220100, 10211100, 12110200, } \\
\text { 20122200, 12011010, 20221010, } 22020210\end{array}$ \\
\hline$A_{3}(8,4,7) \geq 28$ & $2 \times C_{4}$ & $\begin{array}{l}22111110,12122210,11222120,01112111,20222111, \\
11102221,02222221\end{array}$ \\
\hline
\end{tabular}

Table 3: Codes with a nontrivial automorphism group. 


\begin{tabular}{|c|c|c|}
\hline Bound & Group & Orbit Representatives \\
\hline$\overline{A_{3}(9,3,4) \geq 153}$ & $C_{9}$ & $\begin{array}{l}211100000,112200000,101210000,222020000, \\
120120000,221001000,202201000,120011000, \\
102021000,110102000,200212000,210022000, \\
110010100,202010100,200101100,120200200, \\
220010200\end{array}$ \\
\hline$A_{3}(9,3,5) \geq 336$ & $F_{7,3}$ & $\begin{array}{l}112110000,221220000,221100010,112200010, \\
111010010,202220010,122100020,211200020, \\
101110020,222020020,211100001,122200001, \\
202210001,101120001,220200011,110100021, \\
222100002,111200002,211010002,122020002, \\
212000012,101100012,121000022,202200022\end{array}$ \\
\hline$A_{3}(9,3,6) \geq 411$ & $S_{3} \times C_{3}$ & $\begin{array}{l}222111000,221221000,112221000,121220100, \\
122011100,011211100,212021100,021121100, \\
102121100,111022100,022222100,121110200, \\
212110200,111011200,022211200,120221200, \\
202221200,222022200,011222200,220110110, \\
102210110,120101110,011101110,202201110, \\
021202110,210210210,202120210,022220210, \\
220201210,102021210,012202210,220220220\end{array}$ \\
\hline$A_{3}(9,3,7) \geq 405$ & $G^{\prime}$ & $\begin{array}{l}211111100,122111100,112221100,121212100, \\
212212100,111122100,222122100,111211200, \\
222211200,121121200,211222200,122222200, \\
122210110,221220110,210221110\end{array}$ \\
\hline$A_{3}(9,3,8) \geq 171$ & $C_{9}$ & $\begin{array}{l}212111110,221121110,121112110,211222110, \\
122222110,122111210,221211210,112221210, \\
111212210,212122210,222211120,112121120, \\
121221120,211112120,111111220,222121220, \\
211221220,122212220,121122220\end{array}$ \\
\hline$A_{3}(9,4,3) \geq 24$ & $3 \times C_{3}$ & $\begin{array}{l}111000000,002220000,000111000,220000100, \\
002002100,100100200,001010200,010001200, \\
000020110,000000222\end{array}$ \\
\hline$A_{3}(9,4,4) \geq 63$ & $C_{9}$ & $\begin{array}{l}\text { 122100000, 220220000, 201102000, } 210012000 \text {, } \\
101200100,202001100,201010200\end{array}$ \\
\hline
\end{tabular}

Table 3: (Cont.) Codes with a nontrivial automorphism group. 


\begin{tabular}{|c|c|c|}
\hline$\overline{\text { Bound }}$ & Group & Orbit Representatives \\
\hline$A_{3}(9,4,5) \geq 132$ & $C_{8}$ & $\begin{array}{llll}111210000, & 222120000, & 212201000, & 102111000, \\
121102000, & 201222000, & 120110100, & 202210100, \\
220202100, & 201110001, & 110120001, & 101021001, \\
220022001, & 202020201, & 220210002, & 102220002, \\
110011002,202012002,101010102 & \end{array}$ \\
\hline$A_{3}(9,4,7) \geq 120$ & $C_{8}$ & $\begin{array}{llll}222112100, & 122121200, & 211122200, & 121111001, \\
212212001, & 222201101, & 122022101, & 201222101, \\
212021201, & 211221002, & 112122002, & 221210102, \\
111011102, & 202121102, & 120221102, & 112201202\end{array}$ \\
\hline$A_{3}(9,4,8) \geq 56$ & $C_{8}$ & $\begin{array}{l}211121110, \quad 222122210, \quad 212122101, \quad 222111201, \\
121122201,212211102,111222102,121211202\end{array}$ \\
\hline$A_{3}(9,5,5) \geq 36$ & $3 \times C_{3}$ & $\begin{array}{llll}011110100, & 200221100, & 122002100, & 022220200, \\
211001200, & 100112200, & 220010110, & 001202110, \\
110020220, & 002101220,200002211,001010221\end{array}$ \\
\hline$A_{3}(9,5,6) \geq 40$ & $C_{8}$ & $\begin{array}{l}\text { 112022100, } 112101001, \quad 221202001, \quad 101211002, \\
202122002\end{array}$ \\
\hline$A_{3}(9,5,7) \geq 36$ & $3 \times C_{3}$ & $\begin{array}{llll}122122100, & 102111210, & 021222210, & 222201120, \\
111012120, & 221110220, & 110221220, & 201210111, \\
122200211, & 011011211,200122211,012210222\end{array}$ \\
\hline$A_{3}(10,3,5) \geq 522$ & $G^{\prime}$ & $\begin{array}{l}2112100000,1111200000,2221200000,1220110000, \\
2210220000,2121001000,1212001000,1201101000, \\
2022101000,1021011000,2002211000,2202102000, \\
2012022000,1211000001,2122000001,1202100001, \\
2101200001,2220100002,2011100002,1210200002, \\
1021200002,1102200002\end{array}$ \\
\hline$A_{3}(10,3,6) \geq 876$ & $F_{9,4}$ & $\begin{array}{l}1222210000,2111120000,1221120000,1112220000, \\
2120111000,1101221000,0122221000,2220222000, \\
\text { 2101210100, 2202220200, 1112100001, 2221200001, } \\
\text { 2121010001, 2220110001, 0111110001, 1021110001, } \\
\text { 2012110001, 1102210001, 0212210001, 2102120001, } \\
\text { 0222120001, 0121220001, 2022220001, 0102111001, } \\
0201211001,0202222001,2211100002,1222100002, \\
2222010002,1210110002,2101110002,0221110002, \\
0122210002,1202220002\end{array}$ \\
\hline
\end{tabular}

Table 3: (Cont.) Codes with a nontrivial automorphism group. 


\begin{tabular}{|c|c|c|}
\hline Bound & Group & Orbit Representatives \\
\hline$\overline{A_{3}(10,3,7) \geq 960}$ & $F_{9,4}$ & $\begin{array}{l}2121111000,1211211000,222211000,2211121000, \\
1222121000,111122000,2122122000,1212222000, \\
2112110100,2221210100,1212120100,111220100, \\
2122220100,0212111100,022221100,2202122100, \\
2211110001,1222110001,2121210001,2112120001, \\
1110111001,2101121001,0122121001,0211122001, \\
1202122001,2220222001,1101110101,2202220201, \\
1221210002,1211120002,2111220002,1122220002, \\
0221111002,2202111002,1201221002,0212221002\end{array}$ \\
\hline$A_{3}(10,3,8) \geq 760$ & $F_{5,4} \times C_{2}$ & $\begin{array}{l}2112111100,1121121100,222121100,2111221100, \\
1212221100,1211112100,2221212100,2211121200, \\
2122221200,2212212200,2222111010,1122211010, \\
2112121010,2211221010,2121212010,2122101110, \\
2221021110,1112021110,2221102110,2120112110, \\
2022122110,2222201210,1222022210,2220122210, \\
2221222020\end{array}$ \\
\hline$A_{3}(10,3,9) \geq 325$ & $2 \times \mathrm{AGL}_{1}(5)$ & $\begin{array}{l}1111211110,1212221110,2122221110,1211122110, \\
2112122110,1111112210,2222112210,1122122210, \\
2121222210,2212222210,1111222220,1221011111, \\
2121021111,0222221111,1222022111,2120122111, \\
2202122211,0112122211,1012222211,1221022221, \\
1210122221\end{array}$ \\
\hline$A_{3}(10,4,3) \geq 26$ & $3 \times C_{3}$ & $\begin{array}{l}0221000000,0002220000,1100001000,0012001000, \\
0000111000,0200202000,2000002200,0000001110, \\
0000022001,1001000002\end{array}$ \\
\hline$A_{3}(10,4,4) \geq 105$ & $2 \times C_{5}$ & $\begin{array}{l}1021010000,2202010000,0101210000,0122020000, \\
0011120000,1200220000,1100011000,2001021000, \\
0020112000,1010022000,0220010100,2000120100, \\
0000211100,0002022100,2010010200,0002110200, \\
0020220200,0200021200,0000121010,0001012010, \\
0100022020\end{array}$ \\
\hline
\end{tabular}

Table 3: (Cont.) Codes with a nontrivial automorphism group. 


\begin{tabular}{|c|c|c|}
\hline Bound & Group & rbit Representatives \\
\hline$\overline{A_{3}(10,4,5) \geq 204}$ & $2 \times D_{5}$ & $\begin{array}{l}1111100000,222200000,0122110000,0211220000, \\
1101011000,0011111000,0112021000,1020121000, \\
2001221000,2202022000,0022222000,2220010100, \\
0202210100,1201020100,2002120100,0120220100, \\
1010011100,2100021100,0021021100,2001012100, \\
1000222100,1110020200,0101120200,2020022200, \\
2200011010,0100221010,0020120210,0000212210, \\
1100022020,0000121120,0000011111,0000022222\end{array}$ \\
\hline$A_{3}(10,4,6) \geq 420$ & $2 \times \mathrm{AGL}_{1}(5)$ & $\begin{array}{l}2122120000,0121111000,2220211000,1221021000, \\
0212121000,1102221000,1110122000,1110011100, \\
0202211100,2011021100,1200121100,1201012100, \\
2102022100,2020122100,0012222100,2120021200, \\
2002121200,0222022200,0101122200,0220011110, \\
0010121110,0021022110,0100222110,2100012210, \\
0110022220,0200022211\end{array}$ \\
\hline$A_{3}(10,4,7) \geq 380$ & $2 \times \mathrm{AGL}_{1}(5)$ & $\begin{array}{l}1122211000,1212121000,2221222000,2202111100, \\
2211021100,0122121100,1022112100,1112022100, \\
2101122100,1121021200,1011122200,2120021110, \\
\text { 1202021110, 2001221110, 1021022110, 2010122110, } \\
\text { 1100222110, 2100112210, 1001212210, 0220122220, } \\
\text { 0011021111, 0022022211 }\end{array}$ \\
\hline$A_{3}($ & $2 \times$ & $\begin{array}{l}2121221100,2221112100,1211222100,1112221200, \\
1111011110,2222011110,2210221110,1022221110, \\
\text { 1220122110, 0112222110, 1221012210, 2112012210, } \\
0211122210,1102122210,1111022220,2222022220, \\
2012022111,0021222111,1210022211,2101022211\end{array}$ \\
\hline$A_{3}(10,4,9) \geq 120$ & 2 & $\begin{array}{l}1221111110,2112111110,2121222110,2211221210, \\
1111112210,222212210,2222121120,1111221120, \\
1221122220,2112122220,2221021111,1212022111, \\
0112121211,2111022211,1022122121,2021222221\end{array}$ \\
\hline$A_{3}(10,5,5) \geq 72$ & $\gamma_{5}$ & $\begin{array}{l}1111100000,2222200000,1120021000,0012221000, \\
2210012000,0021112000,1202020100,2010120100, \\
0100111100,2101010200,1020210200,0200222200, \\
2000211010,0002012210,1000122020,0001021120\end{array}$ \\
\hline
\end{tabular}

Table 3: (Cont.) Codes with a nontrivial automorphism group. 


\begin{tabular}{|c|c|c|}
\hline$\overline{\text { Bound }}$ & Group & Orbit Representatives \\
\hline$\overline{A_{3}(10,5,6) \geq 75}$ & $C_{10}$ & $\begin{array}{l}2021211000,1111021000,1212202000,1201210100, \\
1120201100,2210022100,2102110200,1022022200\end{array}$ \\
\hline$A_{3}(10,5,7) \geq 120$ & $2 \times D_{5}$ & $\begin{array}{l}1121211000,2201221100,1011112100,1222022100, \\
1112220200,2122021010,1110122010,0110211110, \\
0021121110,2101012110,2202120210,0211022210, \\
0102122120,1001222220,0002221211,2020022211\end{array}$ \\
\hline$A_{3}(10,5,8) \geq 90$ & $2 \times C_{5}$ & $\begin{array}{l}\text { 1211211100, 2122122200, 1222111010, 0211122110, } \\
\text { 1022222110, 2212021210, 1101221210, 1110112210, } \\
2111222020,2220221120,1121012120,2202112120, \\
2011111220,0122211220,2002121111,0110221111, \\
2201022221,0012122221\end{array}$ \\
\hline$A_{3}(10,5,9) \geq 40$ & $2 \times C_{5}$ & $\begin{array}{l}1221212110,2212211210,2121122120,1112121220 \\
0111221111,1102122111,2221022211,2012222221\end{array}$ \\
\hline$A_{3}(10,5,7) \geq 120$ & $2 \times D_{5}$ & $\begin{array}{l}1121211000,2201221100,1011112100,1222022100, \\
1112220200,2122021010,1110122010,0110211110, \\
0021121110,2101012110,2202120210,0211022210, \\
0102122120,1001222220,0002221211,2020022211\end{array}$ \\
\hline$A_{3}(10,5,8) \geq 90$ & $2 \times C_{5}$ & $\begin{array}{l}\text { 1211211100, 2122122200, 1222111010, 0211122110, } \\
\text { 1022222110, 2212021210, 1101221210, 1110112210, } \\
\text { 2111222020, 2220221120, 1121012120, 2202112120, } \\
2011111220,0122211220,2002121111,0110221111, \\
2201022221,0012122221\end{array}$ \\
\hline$A_{3}(10,5,9) \geq 40$ & $2 \times C_{5}$ & $\begin{array}{l}\text { 1221212110, 2212211210, 2121122120, 1112121220, } \\
0111221111,1102122111,2221022211,2012222221\end{array}$ \\
\hline
\end{tabular}

Table 3: (Cont.) Codes with a nontrivial automorphism group.

\begin{tabular}{|c|c|c|}
\hline Group & Order & Generators \\
\hline$\overline{C_{k}}$ & $k$ & $(12 \cdots k)$ \\
\hline$D_{5}$ & 10 & $(12345),(1)(25)(34)$ \\
\hline$S_{3} \times C_{3}$ & 18 & $\begin{array}{l}(123)(456)(789),(147)(258)(369), \\
(1)(23)(4)(56)(7)(89)\end{array}$ \\
\hline $\mathrm{AGL}_{1}(5)$ & 20 & $(12345),(1)(2354)$ \\
\hline$F_{7,3}$ & 21 & $(1234567),(1)(235)(476)$ \\
\hline$G^{\prime}$ (see text) & 27 & $(123456789),(1)(258)(396)(4)(7)$ \\
\hline$F_{9,4}$ & 36 & $(1645)(2987)(3),(1362)(4597)(8)$ \\
\hline$F_{5,4} \times C_{2}$ & 40 & $(12345678910),(1)(28104)(3597)(6)$ \\
\hline
\end{tabular}

Table 4: Groups and generators. 
Exact values:

$\begin{array}{ll}a & d=2 w, \text { Theorem } 7 \\ b & d=2 w-1, \text { Theorem } 8 \\ m & \text { From a Jacobsthal matrix, Theorem } 16 \\ p & \text { Perfect code, Theorem } 17 \\ s & \text { Generalized Steiner system, Theorem } 14 \\ u & \text { Theorem } 15 \\ \text { Lower bounds: } & \\ c & \text { (Quasi-) cyclic code, Section 4.3, Table 3 } \\ g & \text { Special automorphism group, Section 4.3, Table } 3 \\ l & \text { Lexicographic code, Section 4.1, Table 2 } \\ r & \text { Theorem } 5 \\ t & \text { From unrestricted code, Section } 4.2 \\ x & \text { Exhaustive search, Section 3.3, Table } 1 \\ y & \text { Theorem } 9 \\ z & \text { Theorem } 10 \\ \text { Upper bounds: } & \\ j & \text { Theorem } 12 \\ t & \text { From unrestricted code, Section } 4.2 \\ w & \text { Eq. (3) and [25] } \\ x & \text { Exhaustive search, Section 3.3 } \\ y & \text { Theorem } 9 \\ z & \text { Theorem } 10\end{array}$

\section{Acknowledgment}

The authors would like to thank Petteri Kaski for informing them about [9].

\section{References}

[1] E. Agrell, A. Vardy, and K. Zeger, Upper bounds for constant-weight codes, IEEE Trans. Inform. Theory 46 (2000), 2373-2395.

[2] E. Agrell, A. Vardy, and K. Zeger, A table of upper bounds for binary codes, IEEE Trans. Inform. Theory 47 (2001), 3004-3006.

[3] S. Blake-Wilson and K. T. Phelps, Constant weight codes and group divisible designs, Des. Codes Cryptogr. 16 (1999), 11-27.

[4] G. T. Bogdanova, Some ternary lexicographic codes, in: Proc. II International Workshop on Optimal Codes and Related Topics, Sozopol, Bulgaria, 1998, pp. 9-14. 


\begin{tabular}{|c|c|c|c|c|c|c|c|c|c|}
\hline$n$ & $w$ & $d=3$ & $d=4$ & $d=5$ & $d=6$ & $d=7$ & $d=8$ & $d=9$ & $d=10$ \\
\hline 3 & 2 & $b_{b} 3_{b}$ & & & & & & & \\
\hline 4 & 2 & $b^{4} 4_{b}$ & 2 & & & & & & \\
\hline 4 & 3 & ${ }_{s} 8_{s}$ & 2 & & & & & & \\
\hline 5 & 2 & ${ }_{b} 5_{b}$ & 2 & 1 & & & & & \\
\hline 5 & 3 & $u 12_{u}$ & $w^{5} 5_{j}$ & 2 & & & & & \\
\hline 5 & 4 & ${ }_{w} 10_{y}$ & $w^{5} 5_{j}$ & 2 & & & & & \\
\hline$\overline{6}$ & 2 & ${ }_{b} 6_{b}$ & ${ }_{a} 3_{a}$ & 1 & 1 & & & & \\
\hline 6 & 3 & $c 18_{x}$ & ${ }_{c} 8_{z}$ & $b^{4} b$ & 2 & & & & \\
\hline 6 & 4 & $w 30_{y}$ & $w 15_{y}$ & $l^{4} j$ & $r 3_{j}$ & & & & \\
\hline 6 & 5 & $z 24_{y}$ & ${ }_{m} 12_{m}$ & ${ }_{r} 3_{j}$ & 2 & & & & \\
\hline 7 & 2 & ${ }_{b} 7_{b}$ & ${ }_{a} 3_{a}$ & 1 & 1 & 1 & & & \\
\hline 7 & 3 & $s 28_{s}$ & ${ }_{c} 14_{z}$ & ${ }_{b} 4_{b}$ & ${ }_{a} 2_{a}$ & 1 & & & \\
\hline 7 & 4 & ${ }_{c} 60-67_{x}$ & $c 23_{x}$ & $w^{7} x$ & $r 3_{j}$ & 2 & & & \\
\hline 7 & 5 & ${ }_{c} 72-84_{y}$ & ${ }_{c} 32_{t}$ & ${ }_{c} 9_{t}$ & ${ }_{r} 3_{j}$ & 2 & & & \\
\hline 7 & 6 & ${ }_{z} 56_{y}$ & ${ }_{w} 14_{x}$ & $w^{7} 7_{j}$ & 2 & 2 & & & \\
\hline 8 & 2 & $b^{8} b_{b}$ & $a^{4} a_{a}$ & 1 & 1 & 1 & 1 & & \\
\hline 8 & 3 & ${ }_{u} 36_{u}$ & ${ }_{c} 16_{z}$ & ${ }_{b} 5_{b}$ & 2 & 1 & 1 & & \\
\hline 8 & 4 & ${ }_{s} 112_{s}$ & $c^{4} 2_{x}$ & ${ }_{x} 13_{x}$ & ${ }_{w} 5_{j}$ & 2 & 2 & & \\
\hline 8 & 5 & ${ }_{c} 161-214_{z}$ & ${ }_{c} 72-73_{z}$ & ${ }_{x} 18_{x}$ & $w 8_{j}$ & ${ }_{r} 3_{j}$ & 2 & & \\
\hline 8 & 6 & ${ }_{c}^{168-224_{y}}$ & $w^{56} 6_{y}$ & ${ }_{x} 18_{x}$ & $w^{8} 8_{j}$ & ${ }_{r} 3_{j}$ & 2 & & \\
\hline 8 & 7 & ${ }_{p} 128_{p}$ & ${ }_{c} 28_{x}$ & ${ }_{m} 16_{m}$ & $w^{4}{ }_{j}$ & 2 & 2 & & \\
\hline 9 & 2 & ${ }_{b} 9_{b}$ & ${ }_{a} 4_{a}$ & 1 & 1 & 1 & 1 & 1 & \\
\hline 9 & 3 & $s 48_{s}$ & ${ }_{c} 24_{y}$ & ${ }_{b} 6_{b}$ & ${ }_{a} 3_{a}$ & 1 & 1 & 1 & \\
\hline 9 & 4 & ${ }_{c} 153-166_{z}$ & ${ }_{c} 63-72_{z}$ & ${ }_{x} 19_{x}$ & ${ }_{w} 9_{j}$ & ${ }_{b} 3_{b}$ & 2 & 1 & \\
\hline 9 & 5 & $g^{336-403 z}$ & ${ }_{c} 132-151_{z}$ & $c 36_{x}$ & $z 18_{y}$ & $w^{5} 5_{j}$ & $r 3_{j}$ & 2 & \\
\hline 9 & 6 & $g^{411-642_{z}}$ & $w 168_{y}$ & $c^{40-48_{x}}$ & $w 24_{y}$ & ${ }_{w} 6_{j}$ & ${ }_{r} 3_{j}$ & ${ }_{r} 3_{j}$ & \\
\hline 9 & 7 & ${ }_{g}^{405-576 y}$ & ${ }_{c} 120-126_{y}$ & ${ }^{3} 36-38_{x}$ & $z 18_{y}$ & ${ }_{w} 5_{j}$ & ${ }_{r} 3_{j}$ & 2 & \\
\hline 9 & 8 & ${ }_{c} 171-180_{y}$ & ${ }^{5} 56-63_{z}$ & $w 18_{x}$ & ${ }_{w} 9_{j}$ & ${ }_{r} 3_{j}$ & 2 & 2 & \\
\hline 10 & 2 & ${ }_{b} 10_{b}$ & $\bar{a} 5_{a}$ & 1 & $\overline{1}$ & 1 & 1 & 1 & 1 \\
\hline 10 & 3 & ${ }_{s} 60_{s}$ & ${ }_{c} 26_{z}$ & ${ }_{b} 6_{b}$ & ${ }_{a} 3_{a}$ & 1 & 1 & 1 & 1 \\
\hline 10 & 4 & $s 240_{s}$ & ${ }_{c} 105-120_{y}$ & ${ }_{t} 30_{z}$ & ${ }_{w} 15_{j}$ & ${ }_{b} 5_{b}$ & 2 & 1 & 1 \\
\hline 10 & 5 & ${ }_{g} 522-664_{z}$ & $g^{204-288 z}$ & $c 72_{y}$ & ${ }_{t} 36_{y}$ & ${ }_{l} 8_{j}$ & $w^{4} j$ & 2 & 2 \\
\hline 10 & 6 & ${ }_{g}^{8} 876-1343_{z}$ & $g^{420} y$ & $c^{75-120} y$ & ${ }_{t} 60_{y}$ & ${ }_{l} 11_{x}$ & $w^{5}{ }_{j}$ & ${ }_{r} 3_{j}$ & 2 \\
\hline 10 & 7 & ${ }_{g}^{960-1834 z}$ & $g^{380-420 y}$ & $g 120-126_{y}$ & ${ }_{t} 60_{y}$ & ${ }_{x} 13_{t}$ & ${ }_{w} 5_{j}$ & $r 3_{j}$ & 2 \\
\hline 10 & 8 & $g 760-900_{y}$ & $g^{280-315 y}$ & $c 90_{y}$ & $t^{45_{y}}$ & ${ }_{w} 10_{j}$ & $w^{5}{ }_{j}$ & 2 & 2 \\
\hline 10 & 9 & $g 325-400_{y}$ & $g 120-140_{z}$ & $c^{40}$ & ${ }^{2} 20_{m}$ & ${ }_{w} 5_{j}$ & 2 & 2 & 2 \\
\hline
\end{tabular}

Table 5: Bounds on $A_{3}(n, d, w)$ for $n \leq 10$. 
[5] G. T. Bogdanova, A. E. Brouwer, S. N. Kapralov, and P. R. J. Östergård, Errorcorrecting codes over an alphabet of four elements, Des. Codes Cryptogr. 23 (2001), $333-342$.

[6] A. E. Brouwer, H. O. Hämäläinen, P. R. J. Östergård, and N. J. A. Sloane, Bounds on mixed binary/ternary codes, IEEE Trans. Inform. Theory 44 (1998), 140-161.

[7] A. E. Brouwer, J. B. Shearer, N. J. A. Sloane, and W. D. Smith, A new table of constant weight codes, IEEE Trans. Inform. Theory 36 (1990), 1334-1380.

[8] K. Chen, G. Ge, and L. Zhu, Generalized Steiner triple systems with group size five, J. Combin. Des. 7 (1999), 441-452.

[9] J. H. Conway, A. Hulpke, and J. McKay, On transitive permutation groups, LMS J. Comput. Math 1 (1998), 1-8.

[10] T. Etzion, Optimal constant weight codes over $Z_{k}$ and generalized designs, Discrete Math. 169 (1997), 55-82.

[11] F.-W. Fu, T. Kløve, Y. Luo, and V. K. Wei, On the Svanström bound for ternary constant weight codes, IEEE Trans. Inform. Theory 47 (2001), 2061-2064.

[12] F.-W. Fu, A. J. H. Vinck, and S.-Y. Shen, On the constructions of constant-weight codes, IEEE Trans. Inform. Theory 44 (1998), 328-333.

[13] I. S. Honkala and P. R. J. Östergård, Code design, in: E. Aarts and J. K. Lenstra (Eds.), Local Search in Combinatorial Optimization, Wiley, New York, 1997, pp. 441-456.

[14] S. M. Johnson, A new upper bound for error-correcting codes, IRE Trans. Inform. Theory 8 (1962), 203-207.

[15] K. S. Kapralov, The nonexistence of ternary $(10,15,7)$ codes, in: Proc. Seventh International Workshop on Algebraic and Combinatorial Coding Theory (ACCT'2000), Bansko, Bulgaria, 18-24 June, 2000, pp. 189-192.

[16] J. van Lint and L. Tolhuizen, On perfect ternary constant weight codes, Des. Codes Cryptogr. 18 (1999), 231-234.

[17] F. J. MacWilliams and N. J. A. Sloane, The Theory of Error-Correcting Codes, North-Holland, Amsterdam, 1977.

[18] B. D. McKay, nauty user's guide (version 1.5), Computer Science Department, Australian National University, Tech. Rep. TR-CS-90-02, 1990.

[19] S. Niskanen and P. R. J. Östergård, Cliquer user's guide, version 1.0, in preparation. 
[20] P. R. J. Östergård, On binary/ternary error-correcting codes with minimum distance 4, in: M. Fossorier, H. Imai, S. Lin, and A. Poli (Eds.), Applied Algebra, Algebraic Algorithms and Error-Correcting Codes, LNCS 1719, Springer, Berlin, 1999, pp. $472-481$.

[21] P. R. J. Östergård, A new algorithm for the maximum-weight clique problem, Nordic J. Comput. 8 (2001), 424-436.

[22] M. Svanström, A lower bound for ternary constant weight codes, IEEE Trans. Inform. Theory 43 (1997), 1630-1632.

[23] M. Svanström, A class of perfect ternary constant-weight codes, Des. Codes Cryptogr. 18 (1999), 223-229.

[24] M. Svanström, Ternary codes with weight constraints, Ph.D. thesis, Linköpings universitet, Dissertation No. 572, 1999.

[25] M. Svanström, P. R. J. Östergård, and G. T. Bogdanova, Bounds and constructions for ternary constant-composition codes, IEEE Trans. Inform. Theory 48 (2002), $101-111$.

[26] H. Tarnanen, An approach to constant weight and Lee codes by using the methods of association schemes, Ann. Univ. Turku. Ser. A I, No. 182, University of Turku, 1982.

[27] R. J. M. Vaessens, E. H. L. Aarts, and J. H. van Lint, Genetic algorithms in coding theory-A table for $A_{3}(n, d)$, Discrete Appl. Math. 45 (1993), 71-87.

[28] W. D. Wallis, One-Factorizations, Kluwer, Dordrecht, 1997.

[29] J. Yin, Y. Lu, and J. Wang, Maximum distance holey packings and related codes, Sci. China Ser. A, 42 (1999), 1262-1269. 